\title{
ROBEX - Innovative robotic technologies for ocean observations, a deep-sea demonstration mission
}

\author{
F. Wenzhoefer, T. Wulff \\ Alfred-Wegener-Institute Helmholtz Center for Polar and Marine Research \\ D-27570 Bremerhaven, Germany \\ frank.wenzhoefer@awi.de, thorben.wulff@awi.de
}

\author{
S. Floegel, S. Sommer \\ GEOMAR Helmholtz-Center for Ocean Research \\ D- 24148 Kiel, Germany \\ sfloegel@geomar.de, ssommer@geomar.de
}

\author{
C. Waldmann \\ University of Bremen/MARUM \\ D- 24148 Bremen, Germany \\ cwaldmann@marum.de
}

\begin{abstract}
Innovative robotic technologies are a key to study ocean processes in space and time. The work carried out during the ROBEX-Demonstration Mission on RV Polarstern will test the capability of new and innovative technologies, developed during the HGF Alliance ROBEX, in deep-sea environments. Investigations will include Arctic benthic and pelagic ecosystems strongly influenced by climate change, such as marine arctic sediments hosting gas hydrates and arctic deep-sea benthic communities. Different robotic platforms, including 3 types of crawler, glider, AUV, UAVs and senor systems (like Lab-on-aChip and multi- $\mathrm{O}_{2}$-profiler) are described and mission scenarios presented. The use of these new underwater technologies will improve our capabilities to improve our knowledge on the effects of climate change on the Arctic ecosystem and ocean observation.
\end{abstract}

Keywords-Crawler; Glider; Autonomy; Sensor Systems; AUV; UAVs; Deep-Sea

\section{INTRODUCTION}

Our ability to address questions concerning ocean change is fundamentally limited by the lack of key technologies enabling in situ experimentation, conducting targeted sampling, and performing persistent sensor measurements. The joint developments within the HGF Alliance "Robotic Exploration of Extreme Environments" (ROBEX), combining deep-sea and space technology and knowhow, applying risk management methods, advanced robotic technology, and the "simulation first"-approach (common in space projects), opens new ways to study a great variety of deep-sea ecosystems. The major scientific issues of deep-sea research that need to be addressed by novel technological approaches relate to long-term observation of environmental processes, mineral and biological resources, natural hazards, and threats originating from deep ocean processes, detection of biological diversity and specific life forms and adaptation of organisms in extreme environments. The scientific fields outlined above are the main drivers supporting the development of mission-specific advanced infrastructure.

This paper presents the current activities of the HGF Alliance ROBEX in developing innovative technologies and procedures for the exploration of extreme environments and sensor systems for deep-sea observations. We provide an overview on innovative core technologies developed and present mission scenarios for a demonstration mission with $\mathrm{RV}$ Polarstern in the Arctic Ocean (Fig. 1). Within this paper, the planned deep-sea demonstration mission, tackling the challenge of fully autonomous robotic explorations, is described in detail - as is the application of sensor systems covering different spatial and temporal scales. The combination of mobile and stationary platforms and sensor systems enables new ways of ocean observation from surface waters down to the seafloor.

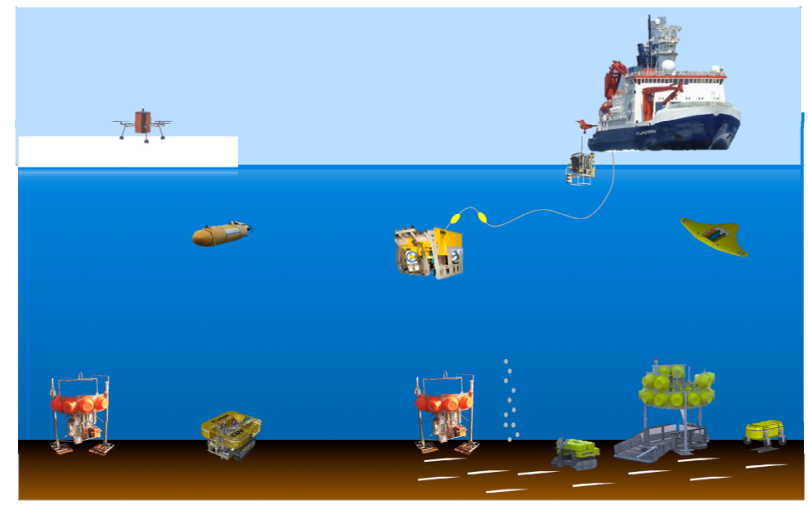

Figure 1. Schematic sketch of the demonstration mission

\section{ROBEX}

The Helmholtz Alliance "Robotic Exploration of Extreme Environments - ROBEX" [1] serves as a collaboration 
platform between explorer from two different worlds - space and deep-sea research. Despite the obviously different physical boundary conditions, like pressure, light and gravity, both environments are facing common challenges for their exploration technologies. Within ROBEX 16 German research institutions, universities and companies cooperate to find solutions for autonomy, navigation, power management and communication. Marine and space scientists and engineers jointly develop technologies to improve our capabilities for the exploration of environments with extreme conditions such as the deep sea, Polar Regions, our Earth's moon and other celestial bodies.

Structured around four work packages for (I) system infrastructure, (II) robotic developments, (III) instrumentation and (IV) scientific challenges ROBEX develops specialized technological systems within specific design teams. Thus it provides strong mutual benefits in the areas of autonomy, mobility \& navigation, manipulation and sensor systems for both the marine and space community (Fig. 2).

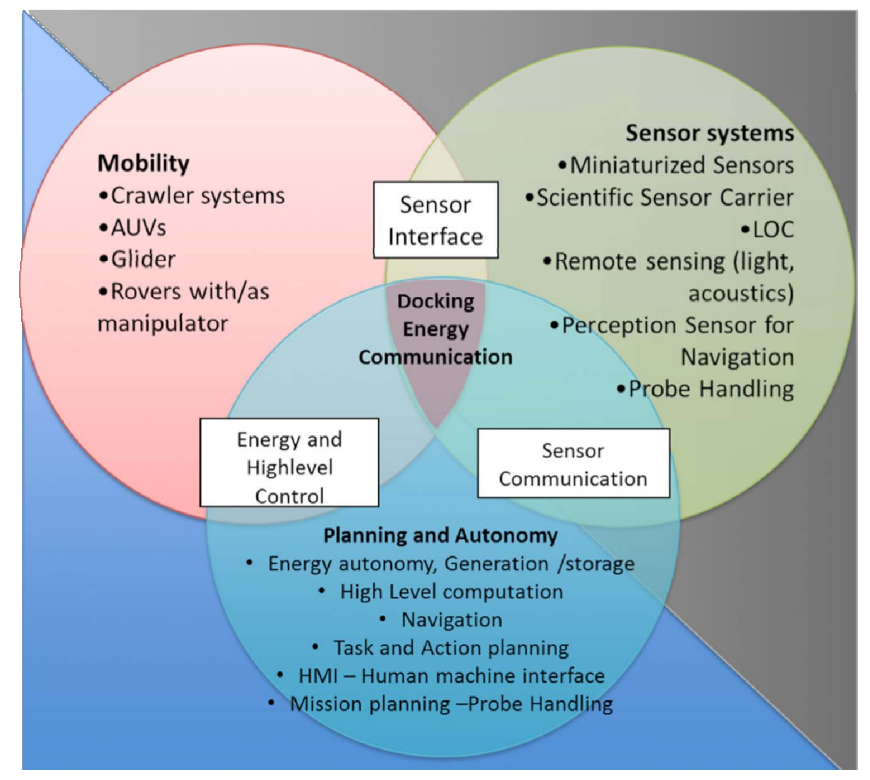

Figure 2. Synergies of the ROBEX fileds of expertiese

During the project representative exploration scenarios for both fields have been defined combining stationary and mobile systems. These demonstration missions shall prove the fundamentally required capabilities of typical explorations mobility, manipulability and autonomy. The overall aim of the deep-sea demonstration mission (Fig. 1) is to demonstrate the applicability of the new and innovative underwater-platforms and sensors for investigating different scientific questions, like the pelagic-benthic coupling in Polar Regions and methane effluxes from the seafloor.

Contrary to the deep-sea demonstration mission, ROBEX cannot perform the space demonstration mission in the real environment, i.e. the Moon. Hence it was necessary to translate and downscale the Moon Mission concept (an Active Seismic Network) into an analogue demonstration mission, which includes the demonstration of the main scientific and technical challenges on a Moon mission (e.g. lander, rover, mobility, communication, deployment, positioning, manipulation).

\section{DeEP SEA Demonstration Mission}

The work carried out during the proposed expedition ROBEX-Demonstration Mission will test the capability of new and innovative technologies, developed during the HGF Alliance ROBEX, in deep-sea environments. Investigations will include Arctic ecosystems strongly influenced by climate change, such as marine arctic sediments hosting gas hydrates and arctic deep-sea benthic communities (Fig. 1). At the gas hydrate stability zone (GHSZ) off Spitzbergen we will quantify gas hydrate deposits as well as water column gas concentrations at different spatial and temporal scales. At the HAUSGARTEN deep-sea observatory in the Fram Strait, studies on the pelagic-benthic coupling will be performed, to investigate how benthic life is governed by the food supply from surface waters. The use of new underwater technologies will thereby improve our capabilities to improve our knowledge on the effects of climate change on the Arctic ecosystem.

During the demonstration mission cruise (PS108, RV Polarstern, 2017) to the Arctic (Fig. 3) the newly developed technologies (Fig. 1), including 3 different types of benthic crawler, each designed for its specific scientific purpose, a glider, unmanned aerial vehicles (UAVs) to support AUV operations at the ice edge, and sensor systems like long-term oxygen profiler, Lab on a Chip (LOC) technology and underwater mass-spectrometer will be used to study biogeochemical processes in the ocean. The work during the expedition will be supported by the ROV Kiel6000 (Geomar, Kiel, Germany).

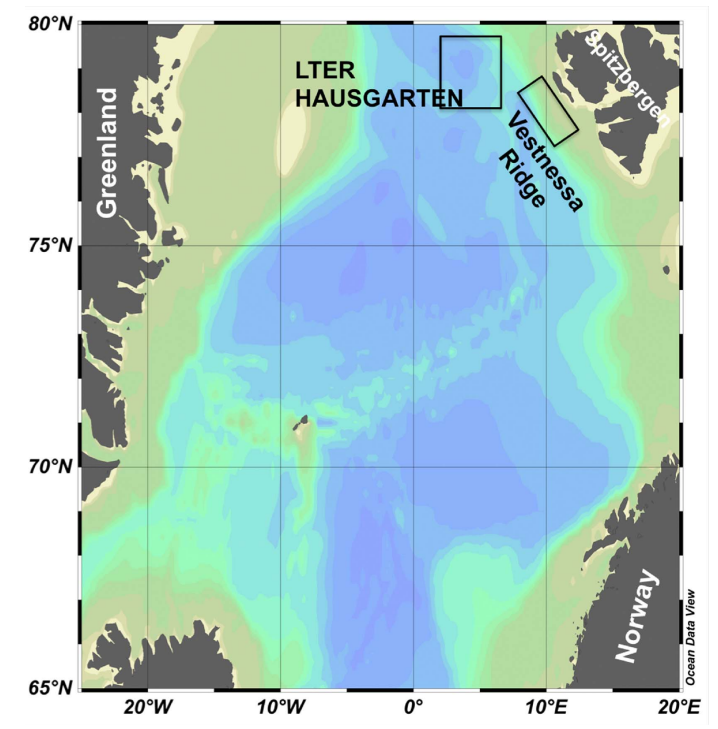

Figure 3. Working areas of the ROXEX-Demonstration Mission cruise PS108 on RV Polarstern

Although there are different needs and strategies for deep-sea missions to investigate the scientific working areas (like vents, 
seeps, hypoxia and under sea ice), a common mobile benthic robotic-system was identified, which can be used at all sites. This benthic robotic-system combines stationary and mobile components, which can be combined as needed. Further such a system combines components for high power capacities, central data storage and transfer with high spatial and temporal flexibility to measure relevant environmental parameters. The major common issue for all deep-sea scenarios (vents, seeps, hypoxia and under sea ice) is to capture the high spatial and temporal variation in (I) environmental parameters (e.g. water chemistry, nutrients), (II) biogeochemical process rates and (III) biological diversity, which requires repeated measurements over larger areas and tidal or seasonal cycles.

Below major key technologies for oceanographic and benthic deep-sea investigations developed or further improved within the ROBEX alliance and operated during the ROBEX Demonstration Mission are described.

\section{A. Crawler CMOVE}

As there has been a common interest in using crawler systems for carrying out seismic measurements on the moon and in the deep sea it had been decided to use a common design and build a group 2-5 identical crawlers (Fig. 4). This system will allow for deploying an array of seismometers at the seafloor based on the previous CMOVE design of MARUM. A number of technical features shall be added which includes an active suspension and a traction control of each individual wheel. With the support of the German Aerospace Agency DLR, Oberpfaffenhofen, a technical implementation for the propulsion system has been selected and is currently under construction.



Figure 4. Sketch of the crawler CMOVE

The planned demonstration will only involve 2 vehicles that will move in a coordinated manner on the seafloor (Fig. 5). The exact position of the system has to be known to be able to properly investigate the collected seismic signals. For that purpose an USBL system operated from board the research vessel will be used. For the field-tests it is expected to operate the system over a duration of a few days.

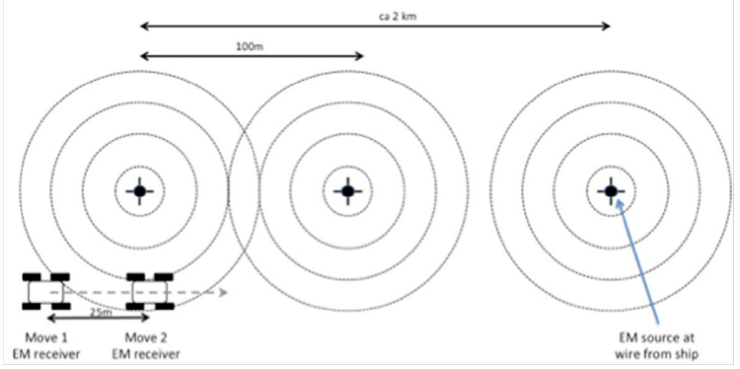

Figure 5. CMOVE-Mission scenario for seismic sudies

\section{B. Crawler MANSIO-VIATOR}

The fully autonomous crawler system MANSIO-VIATOR (latin: harborage-traveller) has been designed and built for long-term benthic deployments and measurements of physical and biogeochemical parameters (Fig. 6). The hangar is used for transport to the site of investigation and for recovery at the ocean surface as well as to recharge the lithium polymer accumulators on the crawler and to transmit data between the two components. The mobile component, a fully autonomously cooperating mobile unit - the crawler is used to repeatedly move along a defined transect to perform various scientific measurements at the sediment-water interface. These include current measurements, $\mathrm{pH}$, oxygen, turbidity, chlorophyll, temperature, salinity, pressure and methane. Simultaneously, a high-resolution 3D-map of the track/area is generated via a structure-from-light/motion approach using a camera and line laser.

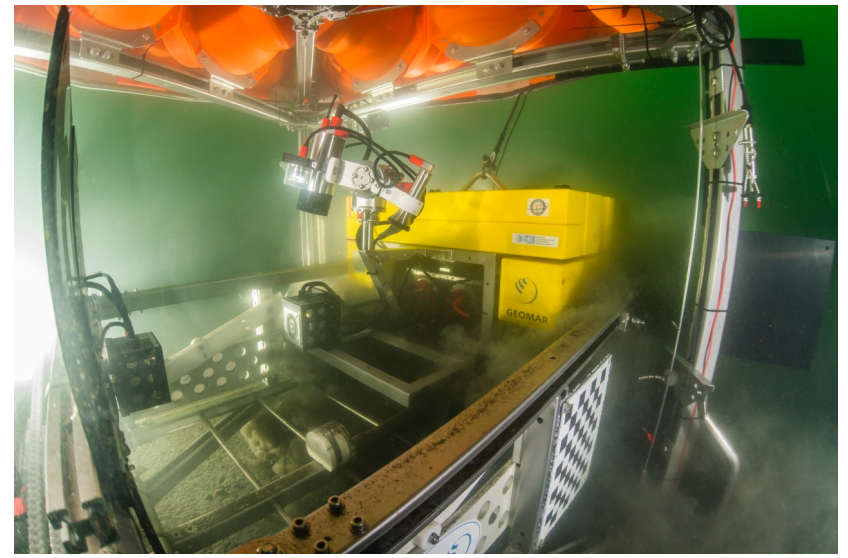

Figure 6. Underwater test of the MANSIO-VIATOR craler lander system.

The advantage of this robotic approach is the capability to cover large distances $(>50-1000 \mathrm{~m})$, which facilitates the coverage of an area considerably larger if compared to fixed benthic observatories. The scientific payload can be adapted in a modular way according to the respective scientific goals. 
During this mission the system will investigate the hydrographic, chemical and biological gradients in time and space within the vicinity of methane seeps at the Vestnessa pockmark region (Fig. 7). The system will be deployed approximately 100 meters from two different seep localities which are characterized by dense microbial mats and pogonophora. This allows the measurement of chemical gradients from the source to natural background bottom water values. The optical survey will additionally cover and map different chemoautotrophic species, normal heterotrophic fauna, i. e. bacterial mats, pogonophora in different densities, and bathyal heterotrophic organisms.

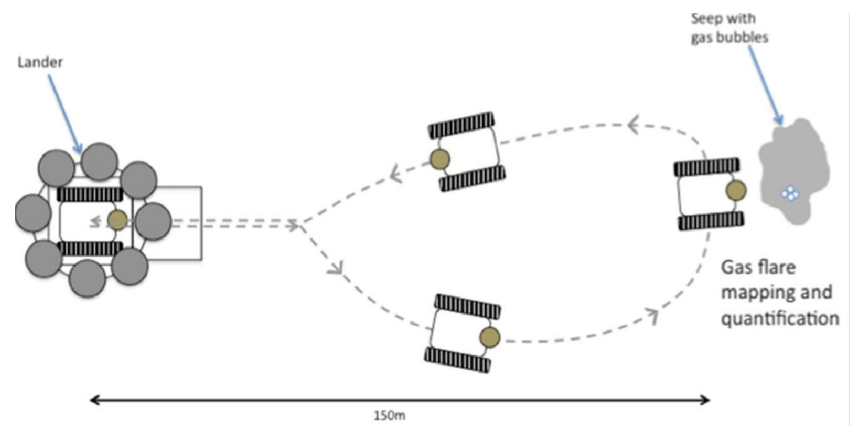

Figure 7. VIATOR-Mission scenario for gas seep investigations

After a video-controlled deployment of the system at the seafloor, the crawler unit leaves the hangar and operates autonomously within a pre-defined radius and returns after completion. After entering the hangar, the crawler will recharge its lithium polymer packs via underwater inductive coupling from lithium cells on the lander.

\section{Crawler TRAMPER}

TRAMPER is a fully autonomous benthic crawler (Fig. 8) capable to perform long-term benthic flux time series measurements at abyssal depth [2]. A lithium-ion battery pack provides the energy to run an oxygen profiling system performing consecutive measurements along its transecting moving on the seafloor. The crawler consists of 4 major components: (I) the basic frame with the caterpillar drive, battery cylinder and acoustic releaser, (II) the scientific payload with a multi-optode profiler, a high-resolution photo camera and an obstacle avoidance camera system, (III) the floatation and (IV) the drop weight.

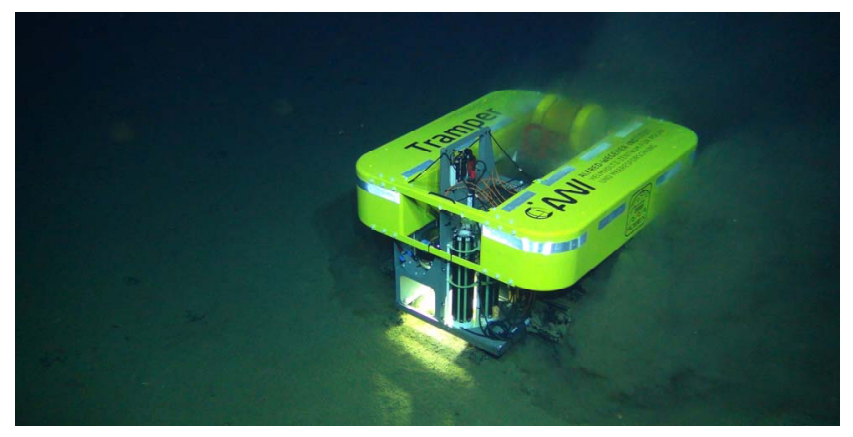

Figure 8. Benthic long-term crawler TRAMPER (Foto: ROV6000, Geomar, Kiel)
A video-guided launching system is used to deploy the crawler at the seafloor. At the seafloor the pre-programmed mission scenario is performed consisting of consecutive sleeping, moving and measurement cycles (Fig. 9). When active Tramper starts with a movement of $15 \mathrm{~m}^{2} 4 \mathrm{~m} \mathrm{~min}^{-1}$ to avoid sediment resuspension. At the new spot an obstacle avoidance camera system takes a photo, which is internally analyzed. In case of identified objects Tramper moves on for another $2 \mathrm{~m}$ to select another measurement spot. This procedure can be repeated for a maximum of 3-times. Then or when the seafloor is free obstacles a high-resolution photo is taken to document the seafloor area before the profiling starts.



Figure 9. TRAMPER long-term mission to study the benthic oxygen consumption over a saisonal cycle

During the measuring cycle 3 optical oxygen sensors are moved vertically in steps of $100 \mu \mathrm{m}$ across the sediment-water interface for a total distance of $25 \mathrm{~cm}$. At each position the sensors equilibrated for $5 \mathrm{sec}$ before the signal is internally stored. After completing the vertical profile the sensors are moved back to the start position and a second high-resolution photo is taken. Afterwards Tramper is sleeping for 164 hours (roughly 7 days) before the next cycle is started (Fig. 9). This operation cycle is in total repeated for at least 52-time to cover a full seasonal cycle. The aim is to cover a seasonal cycle of settling organic matter on the seafloor and to resolve the impact on the benthic community respiration activity.

\section{ROBEX-Glider}

Capitalizing on the know-how in regard to aerospace engineering in the ROBEX consortium it was decided to develop a new type of underwater glider that aims at minimizing the drag while at the same time the payload capacity will be enhanced. With this in mind a so-called blended wing design has been selected as the way forward. Similar designs have been considered in the past and are still under development [3]. However, there is still some ambiguity in regard to the trade-offs that have to be made with this design. As part of ROBEX it is planned to build a prototype and carry out some tests that will deliver better insight into the hydrodynamics of the design, also providing data on the overall performance and finally building a base for future optimization.

The current system is shown in figure 10. The wingspan is $3.4 \mathrm{~m}$ and the overall weight of the hull in air without any interior components is $65 \mathrm{~kg}$.

First test flights in a swimming pool and the test lake of the company Atlas-Elektronik have shown very stable flight behavior so that the system is ready to be equipped with a pneumatic buoyancy engine [4]. Further field tests in the Baltic 
Sea and Mediterranean Sea are planned before the final demonstration in the Arctic from board Polarstern. The final goal for that cruise is to equip the system with some basic scientific instruments and to have the vehicle doing 5-10 dive cycles to demonstrate the capabilities of the new design.

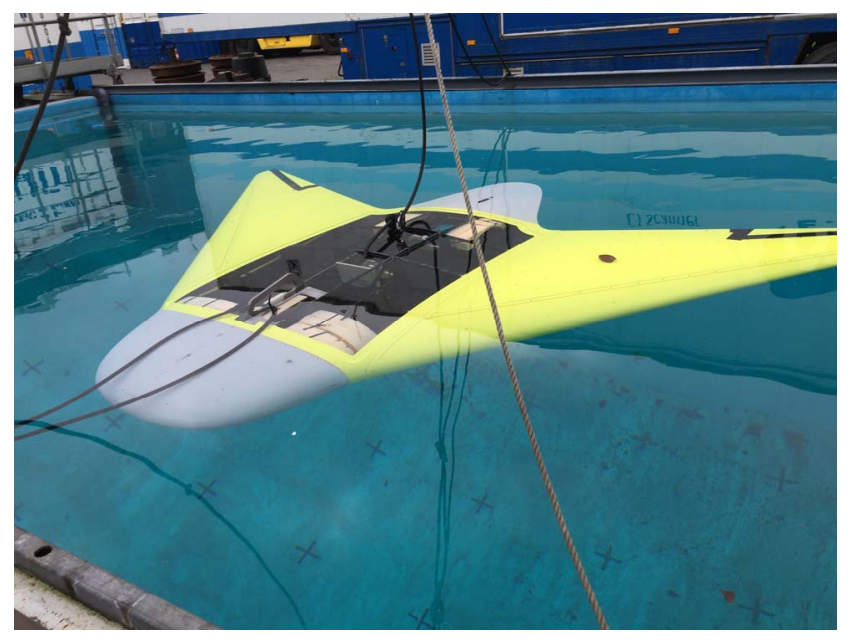

Figure 10. Trimming the weight distribution of the ROBEX glider in the MARUM pool

\section{E. AUV Paul}

The Alfred Wegener Institute (AWI) in Bremerhaven, Germany, has operated its autonomous underwater vehicle (AUV) "PAUL" (Fig. 11) with a special focus on water column studies in high latitudes and especially in the marginal ice zone [5]. PAUL is based on a type 21 vehicle of the American manufacturer Bluefin Robotics (Quincy Massachusetts). The vehicle is $4.3 \mathrm{~m}$ long and its scientific payload encompasses biogeochemical sensors such as a dissolved oxygen or a nitrate sensor as well as a physical oceanography package consisting of a CTD, an upward looking ADCP and a microstructure probe.

Missions in the open water column, with the vehicle operating in a great distance to the seafloor, entail reduced navigation accuracy as its Doppler velocity log (DVL) is normally unable to establish bottom tracking and the vehicle is completely dependent on its inertia navigation system (INS). For this reason, the possibility to use small ship-bound ultra short baseline (USBL) systems to track the vehicle and to correct the navigation errors subsequently, were investigated within ROBEX [6]. The USBL system GAPS of the French manufacturer iXBlue (Marly le Roi, France) was used during testing.

After a dive in the open water column, two data sets are available which both contain errors. The AUV navigation data are consistent yet contain a certain drift. The GAPS data are normally inconsistent and outliers have to be removed yet they represent the only available independent source. In a stepwise process encompassing four corrective phases these two datasets are merged to statistically reconstruct the real dive path of the vehicle.

For phase one, a constant spatial drift of the INS is assumed. Taking a vehicle which has just surfaced, the difference between the last INS-determined position and the first GPS-determined position roughly represents orientation and extent of the spatial drift the INS experienced during its time submerged. As a linearly increasing error is assumed, the AUV's navigation data can be corrected accordingly.

In the second phase, the algorithm removes outliers from the GAPS data. Detailed analyses indicated that unrealistic GAPS position estimates (Lat + Lon) correlate with unrealistic depth estimates. Thus, if the difference between the GAPS depth and the vehicle depth exceeds a certain tolerance, the respective position estimates are disregarded.

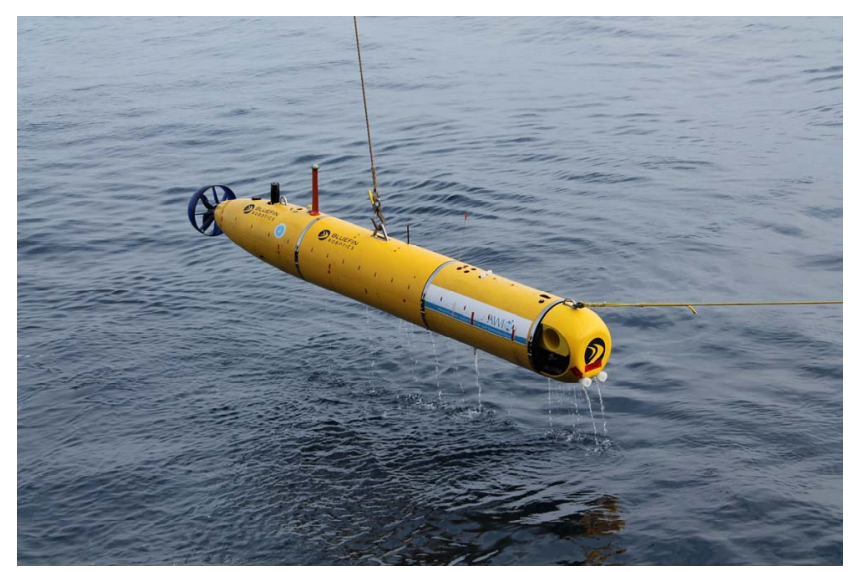

Figure 11. AUV PAUL is being recovered after completing a dive in the Arctic Ocean

In phase three, the remaining GAPS dataset is further reduced by identifying position estimates which most likely represent true locations of the vehicle. Here, it is the basic assumption that the longer a period of consecutive, realistic tracking results (from phase two) is not interrupted by a "loss of tracking" event, the more reliable the position estimate can be considered. For this reason, the algorithm identifies sequences of consecutive, realistic GAPS tracking results with a certain minimal number of elements. Using these sequences of consecutive estimates, a weighted average is calculated pushing the calculated position towards the reliable end of the sequence. Following the naming convention for spline functions, the calculated positions are further referred to as "knots".

In the fourth and final phase, the algorithm the AUV data corrected during phase one are merged with the knots of phase three.

The method reconstructs the dive path (Fig 12) of the AUV to provide resilient georeferencing of the collected data. Although there is some uncertainty remaining as for every statistical method the algorithm represents a successful attempt to minimize the spatial uncertainty of AUV water column studies. Especially in the marginal ice zone, featuring steep 
gradients for various parameters, the algorithm proofed its value and is meanwhile applied by default at AWI.



Figure 12. Chart of one of PAUL's under ice dives. Raw AUV navigation data are indicated as a red line. White dots represent raw GAPS tracking results. The broken grey lines illustrate the drift of the ice edge for the time of the dive

\section{F. $U A V S$}

Starting in 2011 and 2012, there were first tests of operating unmanned aerial vehicles (UAVs) in high latitudes and fly them from research vessels in order to land them on the ice. As these UAVs were still remotely controlled, their operational range was limited to the pilot's visual range. An additional constraint was the magnetic field at high latitude as commercial systems depend on a compass to determine their heading. The horizontal component of the earth's magnetic field is weak at high latitudes and the field is distracted by the ship. However, especially in 2012, during Arctic expedition ARK 27/2 (RV Polarstern), UAVs proofed to be valuable instruments [7].

As a consequence, within ROBEX it is intended to develop necessary technologies for autonomous flight missions and to provide the UAVs with long-range capabilities $(50 \mathrm{~km}+)$. Two different types of UAVs are currently under development.

The landing UAV, developed by the University of Wuerzburg, is a quadrocopter of about $60 \mathrm{~cm}$ diameter that will fly from the ship to the ice. Cameras, thermal sensors and long range radio transmission using tracking antennas will help the human pilot to determine a suitable landing spot on the ice. Normal deployment times of the landing UAV on the ice are expected to be up to 6 hours. After that, the vehicle will begin an autonomous return flight to the ship. Completing first Arctic tests in summer 2015 the vehicle was largely modified. The current version of the UAV accomplished its maiden flight in early 2016 (Fig. 13). As of July 2016, the UAV sees extensive testing to optimize the heading determination based on carrierphase differential GPS. Crucial parts of the UAV, such as motors, thermal sensors, batteries, were tested during Arctic expedition PS 99.2 (RV Polarstern).

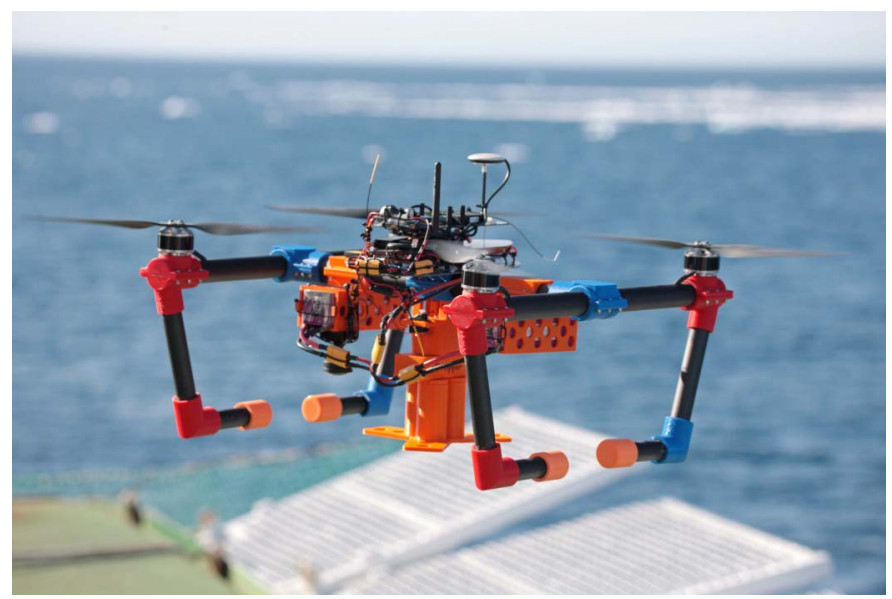

Figure 13. The quad-rotor landing UAV in take-off from the RV Polarstern

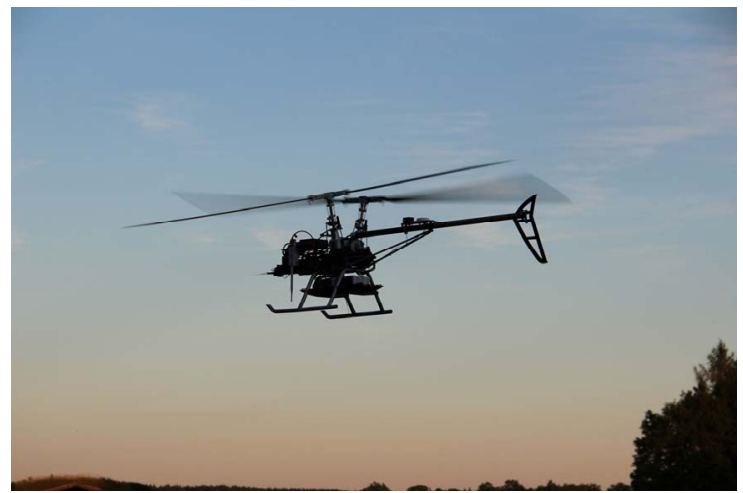

Figure 14. The twin-rotor exploration UAV during a test flight in 2016

The exploration UAV, developed by the German aerospace agency (DLR) is an electric twin rotor helicopter with rotors of about $2.5 \mathrm{~m}$ in diameter (Fig. 14). With a take off mass of 25 $\mathrm{kg}$ it is able to stay airborne for up to $40 \mathrm{~min}$ and reaches a cruise speed of up to $70 \mathrm{~km} / \mathrm{h}$. The vehicle will carry cameras to create a big scale photo-mosaic of the ice surface and it is supposed to conduct its missions completely autonomous including the landing on the ship. A guiding system working with radio beacons to provide the UAV with exact motion data of the ship was successfully tested in 2015. The autonomous approach of the UAV was simulated with the instruments being moved by the ship's crane. As of July 2016, the vehicle is in its final assembly stage. After first tests in Germany, the vehicle will be deployed in a first field campaign in South America in 2016. The exploration UAV can be also used as range extender for landing UAV. The landing UAV could be 
automatically deployed by the exploration UAV on the ice and has only to fly one way back from the ice to the ship.

\section{G. Sensor systems}

In marine and space sciences, a strong need towards in situ measurements on various temporal and spatial scales exists. Ocean sciences for example would profit from intelligent sensor networks (observatories) that are able to capture seasonal variability of important environmental parameters such as oxygen, gases and nutrients in relation to their physical environment (e.g. currents, internal waves). This is of utmost importance to better understand seafloor release of greenhouse gases, ocean acidification or the spread of anoxia and to assess the consequences for the overall ecosystem services and regional economies. General requirements of sensor systems encompass:

i. their strict miniaturization and combination of different sensors in one system enabling the simultaneous measurement of various parameters

ii. the establishment of sensor networks or sensor swarms allowing synoptic measurements covering a wider spatial scale and over longer time periods

iii. sensor implementation on different carriers (e.g. gliders, crawlers, floats, etc.), which demands for low weight, low energy consumption, high signal stability, in situ calibration, and mechanical robustness

iv. a high degree of autonomy of sensors. Autonomy comprises automated sampling including the precise and possibly repeated positioning of probes in the water column, in soils or sediments, sampling preparation and analysis.

Beside other sensor systems such as optical sensors, underwater mass spectrometry for the simultaneous measurements of gases [8], Lab on a chip technology (LOC) is applied within ROBEX to address the above outlined challenges.

The miniaturisation of standard laboratory procedures down to "chip size" is rapidly progressing in medicine and biological/pharmaceutical industries but is still in its infancy in marine sciences [9], [10]. LOC technology offers a broad range of applications ranging from chemical analytics, miniaturized bioreactors for the synthesis of e.g. biomolecules, to cellular assays and experiments. Obvious advantages of LOCs include their small size, low weight, low energy demand, low sample and reagent volumes, and fast processing times, which render them particularly suitable for underwater deployment on a variety of different vehicles such as crawlers, AUVs, landers or gliders. First nitrate and nitrite time series measurements close to the seafloor in $170 \mathrm{~m}$ water depth using self-calibrating LOCs that are based on the design by [11] offshore Mauritania has proven the robustness and reliability of this technology in underwater application [12]. During the demonstration mission a LOC for the measurement of dissolved iron will be applied (Fig. 15). The design of this LOC is a modified version of the LOC that already has been successfully used to measure iron along oxic to anoxic gradients in the Gotland Basin (Baltic Sea) [13]. The chip will be implemented on towed systems as well as on the crawler VIATOR together with other sensor packages.

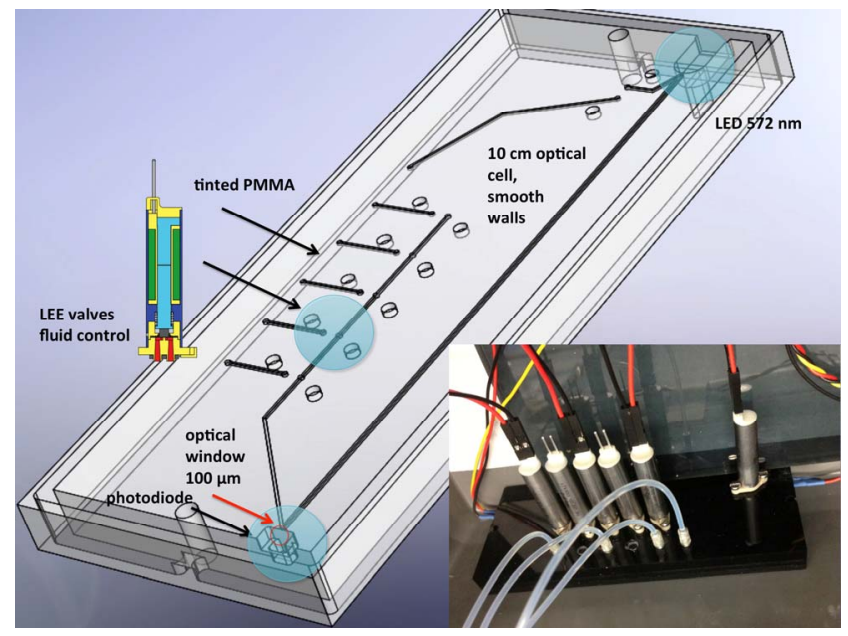

Figure 15. CAD drawing of the microfluidic chip showing microfluidic channels, location of the LED and photodiode for the spectrophotometric Fe measurement, valve ports and mounting holes for Lee valves (www.leeproducts.co.uk). Inset shows a photo of the chip made of tinted PMMA. Chip design modified after [13].

Long-term studies of chemical components require robust, long living, stable and/or an appropriate number of sensors. A new in situ system to monitor sediment oxygen distributions year-round with multiple, needle-type oxygen optodes has been developed (Fig. 16). Mounted on a mobile autonomous crawler the multi-optode-profiler carrying 18 optodes separated in 3 revolver magazines allows a successive use of the sensors during the deployment [2]. A novel in situ calibration-system that provides recurrent zero-point calibrations in situ throughout the deployment is added to validate the stability of the sensor signal and to ensure long-term accuracy.

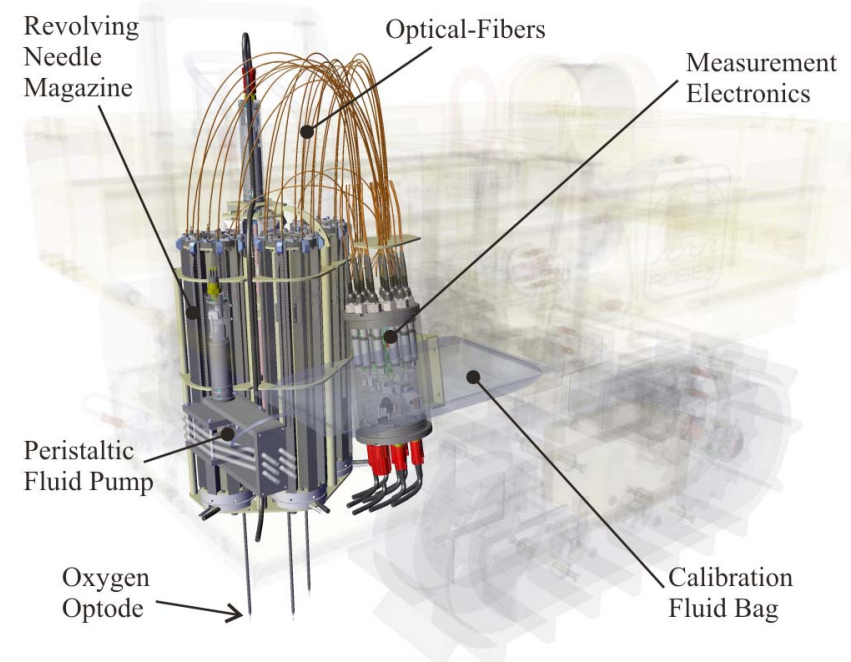

Figure 16. Multi-oxygen profiler 


\section{FUTURE PERSPECTIVES}

In a following step stationary or mobile robotic platforms, either autonomous or tele-operated, shall be interconnected. This includes underwater communication to operate these systems in swarms and event-triggered missions but also interconnection via satellites. For such underwater platforms novel, strongly miniaturized and robust biological and biogeochemical sensing systems will become necessary. These sensor systems require a high degree of autonomy with regard to taking the samples, sample processing and detection and should further encompass the capability to conduct in situ experiments. Another future direction should address the communication between vehicles carrying sensors and the sensor package. Via cybernetic feedback loops this will enable for chemical mapping, sampling along gradients and event triggered measurements based on a self-organizing sensing network.

\section{ACKNOWLEDGMENT}

We strongly appreciate the contribution and cooperation of all ROBEX partners and members. This work is supported by the Helmholtz Association - HGF-Alliance ROBEX "Robotic Exploration of Extreme Environments" (contract number HA-304).

\section{REFERENCES}

[1] ROBEX - HGF Alliance "Robotic Exploration of Extreme Environments" http://www.robex-allianz.de/

[2] Wenzhoefer F, Lemburg J, Hofbauer M, Lehmenhecker S, Faerber P (2016) "Tramper - An autonomous crawler for long-term benthic oxygen flux studies in remote deep-sea ecosystems," Oceans16/IEEE, Monteray, USA, September 2016.
[3] Hildebrand, J., D’Spain, G., http://www.onr.navy.mil/reports/FY12/ mbhilde1.pdf.

[4] Waldmann, C. et al., (2016) Performance data of a pneumatic variable buoyancy engine for a newly designed underwater glider, IEEE OCEANS, Monterey 2016.

[5] Wulff, T., Lehmenhecker, S., Bauerfeind, E., Hoge, U., Shurn, K., Klages, M. (2013). Biogeochemical research with an Autonomous Underwater Vehicle: Payload structure and arctic operations. In OCEANS-Bergen, 2013 MTS/IEEE (pp. 1-10). IEEE.

[6] Wulff, U., Wulff, T. (2015). Correcting Navigation Data of ShallowDiving AUV in Arctic. Sea Technology, 56(3), 27-30.

[7] Lehmenhecker, S., Wulff, T. (2013). Flying Drone for AUV Under-Ice Mission. Sea Technology, 54(2), 61-64

[8] Sommer S, Schmidt M, Linke P (2015) Continuous inline mapping of a dissolved methane plume at a blowout site in the Central North Sea UK using a membrane inlet mass spectrometer - Water column stratification impedes immediate methane release into the atmosphere. Marine Petroleum Geology, 68, 766-775.

[9] Mark D, Haeberle S, Roth G, von Stetten F (2010) Microfluidic lab-ona-chip platforms: requirements, characteristics and applications. Chem. Soc. Rev., 39, 1153-1182.

[10] Whitesides GM (2006) The origin and the future of microfluidfics. Nature, 442, doi:10.1038/nature05058.

[11] Beaton AD, Cardwell CL, Thomas RS, Sieben VJ, Legiret F-E. Waugh $\mathrm{EM}$, et al. (2012) Lab-on-chip measurement of nitrate and nitrite for in situ analysis of natural waters. Environ. Sci. Technol., 46: 9548-9556.

[12] Yücel et al., 2015 Yücel M, Beaton AD, Dengler M, Mowlem MC, Sohl F, Sommer S (2015) Nitrate and nitrite variability at the seafloor of an oxygen minimum zone revealed by a novel microfluidic in situ chemical sensor. PLOS ONE, DOI:10.1371/journal.pone.0132785.

[13] Milani A, Statham PJ, Mowlem MC, Conelly DP (2015) Development and application of a microfluidic in situ analyzer for dissolved $\mathrm{Fe}$ and $\mathrm{Mn}$ in natural waters. Talanta 136, 15-22, doi.org/10.1016/j.talanta.2014.12.045. 